\title{
Inhibiting NFAT1 for breast cancer therapy: New insights into the mechanism of action of MDM2 inhibitor JapA
}

\author{
Jiang-Jiang Qin ${ }^{1,2, *}$, Wei Wang ${ }^{1,2, *}$, Sukesh Voruganti ${ }^{1}$, Hui Wang ${ }^{3}$, Wei-Dong Zhang ${ }^{4}$, \\ Ruiwen Zhang ${ }^{1,2}$ \\ ${ }^{1}$ Department of Pharmaceutical Sciences, School of Pharmacy, Texas Tech University Health Sciences Center, Amarillo, TX, USA \\ ${ }^{2}$ Cancer Biology Center, School of Pharmacy, Texas Tech University Health Sciences Center, Amarillo, TX, USA \\ ${ }^{3}$ Institute for Nutritional Sciences, Shanghai Institutes for Biological Sciences, Chinese Academy of Sciences, Shanghai, PR China \\ ${ }^{4}$ School of Pharmacy, Shanghai Jiao Tong University, Shanghai, PR China \\ *These authors contributed to this work equally
}

Correspondence to:

Ruiwen Zhang, e-mail: ruiwen.zhang@ttuhsc.edu

Wei Wang, e-mail: wwei.wang@ttuhsc.edu

Keywords: JapA, NFAT1, MDM2, p53, breast cancer

Received: June 15,2015 Accepted: September 28, $2015 \quad$ Published: October 08, 2015

\section{ABSTRACT}

Transcription factor NFAT1 has been recently identified as a new regulator of the MDM2 oncogene. Targeting the NFAT1-MDM2 pathway represents a novel approach to cancer therapy. We have recently identified a natural product MDM2 inhibitor, termed JapA. As a specific and potent MDM2 inhibitor, JapA inhibits MDM2 at transcriptional and posttranslational levels. However, the molecular mechanism remains to be fully elucidated for its inhibitory effects on MDM2 transcription. Herein, we reported that JapA inhibited NFAT1 and NFAT1-mediated MDM2 transcription, which contributed to the anticancer activity of JapA. Its effects on the expression and activity of NFAT1 were examined in various breast cancer cell lines in vitro and in MCF-7 and MDA-MB-231 xenograft tumors in vivo. The specificity of JapA in targeting NFAT1 and NFAT1-MDM2 pathway and the importance of NFAT1 inhibition in JapA's anticancer activity were demonstrated using NFAT1 overexpression and knockdown cell lines and the pharmacological activators and inhibitors of NFAT1 signaling. Our results indicated that JapA inhibited NFAT1 signaling in breast cancer cells in vitro and in vivo, which plays a pivotal role in its anticancer activity. JapA inhibited the nuclear localization of NFAT1, disrupted the NFAT1-MDM2 P2 promoter complex, and induced NFAT1 proteasomal degradation, resulting in the repression of MDM2 transcription. In conclusion, JapA is a novel NFAT1 inhibitor and the NFAT1 inhibition is responsible for the JapA-induced repression of MDM2 transcription, contributing to its anticancer activity. The results may pave an avenue for validating the NFAT1-MDM2 pathway as a novel molecular target for cancer therapy.

\section{INTRODUCTION}

Oncogene addiction demonstrates the dependence of cancer cells on a single or few activated oncogenes for their survival, which has been supported by the accumulating evidence from preclinical and clinical studies [1-2]. Therefore, treating the specific type of cancer via the inhibition of corresponding oncogenes can lead to dramatic response and improved survival of patients with cancer [2]. The mouse double minute 2 (MDM2) oncogene is amplified and overexpressed in various human cancers and plays an important role in cancer development and progression via 553 -dependent and $\mathrm{p} 53$-independent mechanisms of action [3-7]. MDM2 has been demonstrated as a promising molecular target for cancer therapy and many MDM2 inhibitors have been developed as new anticancer drugs [8-15]. In an effort to screen small molecule inhibitors of MDM2 for the development of novel and effective cancer therapeutics, we identified a potent and selective MDM2 inhibitor, termed JapA (Figure 1A) [13].

JapA is a structurally unique dimeric sesquiterpenoid that was isolated from the aerial part of Inula japonica Thunb, a plant that has been used in traditional Chinese 
A

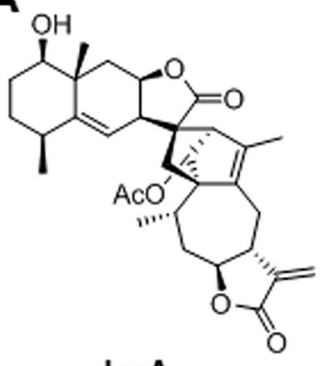
JapA
B

Normal Breast Cancer Cell Lines C

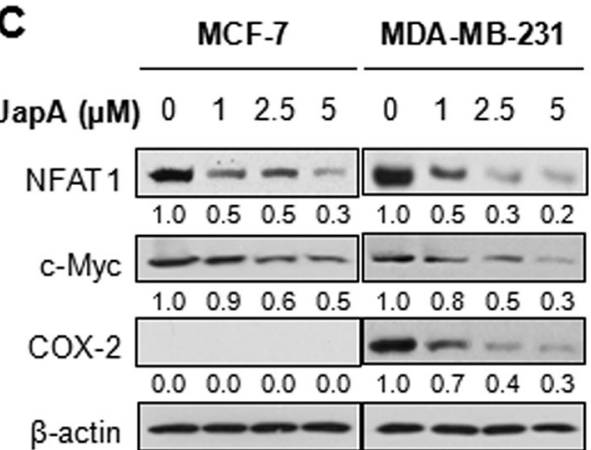

MDA-MB-231 Tumors
D

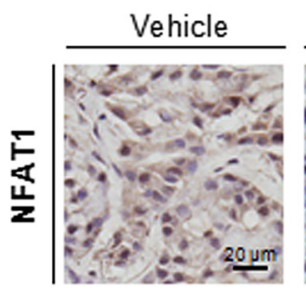

MCF-7 Tumors
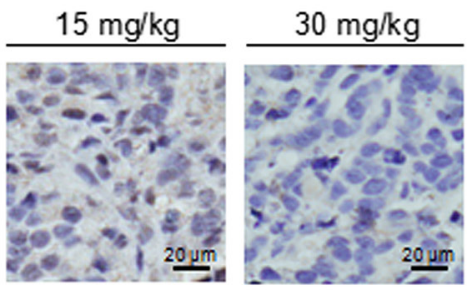

E

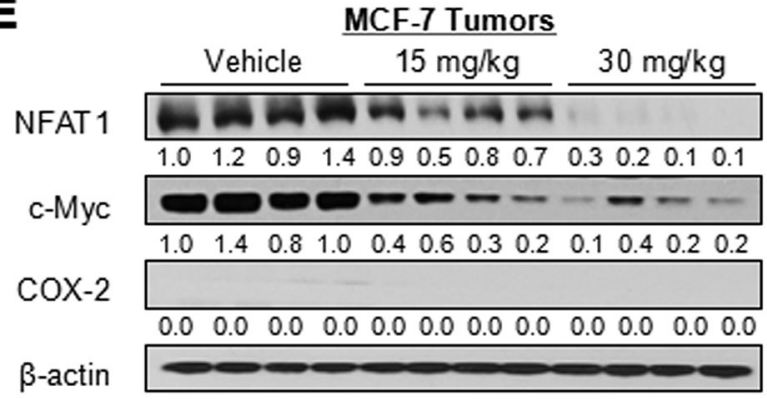

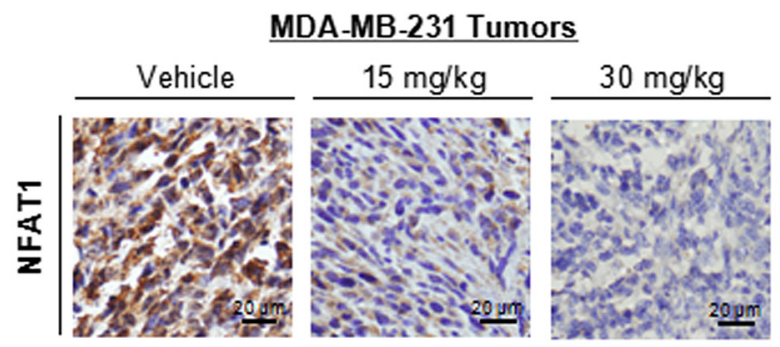

MDA-MB-231 Tumors

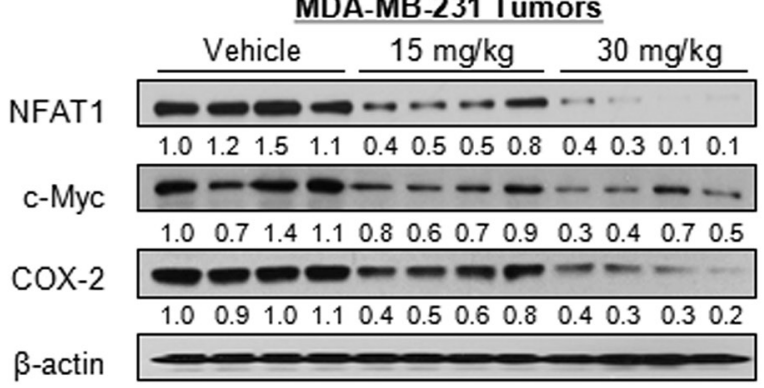

Figure 1: Effects of JapA on NFAT1 signaling pathway in breast cancer cells in vitro and in vivo. A. The chemical structure of JapA. B. Human breast epithelial cells and breast cancer cells were exposed to $2 \mu \mathrm{M}$ JapA for $24 \mathrm{~h}$. The protein level of NFAT1 was detected by western blotting. C. MCF-7 and MDA-MB-231 cells were exposed to various concentrations of JapA for $24 \mathrm{~h}$ for the protein expression of NFAT1, c-Myc and COX-2. The experiments were repeated three times. D, E. JapA was administered by i.p. injection (15 or $30 \mathrm{mg} / \mathrm{kg} / \mathrm{d}, 5 \mathrm{~d} / \mathrm{wk}$ ) to nude mice bearing MCF-7 or MDA-MB-231 xenograft tumors. After 5 weeks (MCF-7) or 3 weeks (MDA-MB-231) JapA treatment, the tumor tissues were analyzed for (D) the protein expression of NFAT1 by immunohistochemistry (scale bar, $20 \mu \mathrm{m}$ ) and (E) the protein expression of NFAT1, c-Myc and COX-2 by Western blotting (each lane represents a different tumor sample). The intensity ratio under each band was obtained by IMAGEJ software analysis normalized on untreated control.

medicine for the treatment of inflammation, diabetes, digestive disorders, and bronchitis [16-18]. JapA has the similar structural features of artemisinin and parthenolide, which are under preclinical and clinical studies for cancer therapy [19]. However, due to its dimerization status and unique mechanisms of action, JapA is considered to be more effective than these analogs as an anticancer drug. We have demonstrated that JapA inhibits the tumor growth and prevents metastasis in breast cancer xenograft models, without inducing any host toxicity [13]. It has also been observed that JapA is safe and effective in treating other human cancers containing high expression levels of MDM2, e.g., Burkitt lymphoma and lung cancer [20-21]. Mechanistically, JapA decreases MDM2 protein stability and inhibits $M D M 2$ transcription, regardless of p53 status of the cells or tumors [13]. At the post-translational level,
JapA directly binds to MDM2 protein and induces MDM2 auto-ubiquitination and proteasomal degradation. JapA has also been found to inhibit $M D M 2$ transcription in a nuclear factor of activated T cells (NFAT)-dependent manner, but the molecular mechanism is still not clear yet.

NFAT is a group of inducible transcription factors with five distinct family members NFAT1 to NFAT5, and it has been demonstrated to play crucial roles in the regulation of various aspects of the immune system and numerous developmental programs in vertebrates (reviewed in references [22-23]). The NFAT proteins regulate diverse cellular functions, such as cell survival, cell cycle progression, migration, invasion, and angiogenesis [24-25]. Increasing evidence suggests the dual roles for NFAT isoforms as oncogene and tumor suppressor in different types of human cancer [24, 26]. 
NFAT1, the first identified member of NFAT family is overexpressed and constitutively activated in several human cancers, including breast cancer [27-30]. NFAT1 is involved in the tumor growth and metastasis through regulating the expression of its target genes, e.g., c-Myc and Cyclooxygenase 2 (COX-2) [24-26]. We have recently discovered that NFAT1 activates the MDM2 oncogene and this pathway contributes to the overexpression of MDM2 in cancer cells with non-functional p53 [31]. Therefore, targeting NFAT1 and NFAT1-MDM2 pathway could be a promising strategy for the discovery of novel cancer therapeutic agents.

The present study was designed to investigate the molecular mechanisms for NFAT1-mediated inhibitory effects of JapA on MDM2 transcription and to demonstrate the role of NFAT1 in JapA's anticancer activity in vitro and in vivo. Since the initial evidence for JapA-induced MDM2 inhibition was obtained with in vitro and in vivo breast cancer models [13], we utilized the same models in the present study. Our results not only helped elucidate the molecular mechanism of JapA as a new class of NFAT1 inhibitor, but also would facilitate the validation of the therapeutic potential of targeting NFAT1 and NFAT1MDM2 pathway, providing a basis for further preclinical and clinical development of NFAT1-MDM2 inhibitors for human cancer therapy.

\section{RESULTS}

\section{JapA inhibits NFAT1 signaling in breast cancer cells in vitro and in vivo}

JapA has been found to inhibit the MDM2 transcription in an NFAT-dependent manner, while NFAT1 has been recently identified as a novel activator of the MDM2 oncogene [13, 31]. Therefore, we examined whether JapA (Figure 1A) affects NFAT1 expression in human normal breast cells and breast cancer cells. As shown in Figure 1B, a significant inhibition of NFAT1 expression by JapA was observed in MCF-7 (p53 wildtype), MCF-7/p53 ${ }^{-/}$(p53 knockdown), MDA-MB-231 (p53 mutant), and MDA-MB-468 (p53 mutant) human breast cancer cell lines. There was no apparent reduction of NFAT1 expression levels in human breast epithelial MCF$10 \mathrm{~A}$ and human mammary luminal epithelial (HMLE) cell lines. We further demonstrated that JapA inhibited the protein expression of NFAT1 and its transcriptional responsive genes c-Myc and $\mathrm{COX}-2$ in a concentrationdependent manner in both the MCF-7 and MDA-MB-231 cell lines (Figure 1C).

The effects of JapA on the NFAT1 signaling were examined in the same breast cancer xenograft tumors we used in the previous study [13]. The significant downregulation of MDM2 expression levels by JapA has been observed in these tumor samples. Compared with vehicle-treated tumors, JapA reduced the expression levels of NFAT1 in both the MCF-7 and MDAMB-231 breast cancer xenograft models, as detected by immunohistochemical staining (Figure 1D) and western blotting (Figure 1E). Consistent with the in vitro results, JapA treatment also reduced the protein levels of c-Myc and COX-2 in the tumors (Figure 1E). Together, these results suggested that JapA inhibits NFAT1 signaling in breast cancer cells in vitro and in vivo.

\section{JapA inhibits NFAT1-mediated MDM2 protein expression}

Considering that NFAT1 upregulates MDM2 expression in a p53-independent manner [31], we examined the effects of JapA on NFAT1-mediated MDM2 expression. As shown in Figure 2A, JapA decreased the MDM2 protein expression in both MCF-7 and MDAMB-231 cell lines, regardless of p53 status of the cells. However, the enforced expression of NFAT1 induced MDM2 protein expression and reduced the inhibition of MDM2 by JapA. Similar results were observed in breast cancer cells transfected with or without HA-tagged constitutively-activated NFAT1 (CA-NFAT1) followed by JapA treatment (Figure 2B). In contrast, the transfection of a dominant-negative NFAT (DN-NFAT) (Figure 2C) or NFAT1 siRNA (Figure 2D) led to the downregulation of MDM2 expression, which was further enhanced by JapA in both breast cancer cell lines. These results indicated an inhibitory effect of JapA on NFAT1-activated MDM2 protein expression.

\section{JapA represses NFAT1-mediated MDM2 transcription}

We further examined the effects of JapA on NFAT1mediated $M D M 2$ transcription. As shown in Figure 3A, HA-NFAT1 overexpression increased the MDM2 mRNA levels $(P<0.01)$, and JapA reduced both the basic and HA-NFAT1-induced MDM2 mRNA expression $(P<0.05)$ in both MCF-7 and MDA-MB-231 cell lines. However, JapA treatment had no significant effects on the DNNFAT-attenuated MDM2 mRNA expression in these cells (Figure 3B). Similarly, JapA reduced the MDM2 mRNA expression that was induced by ionomycin (ION, an activator of calcineurin) (Figure $3 \mathrm{C}$ ), but it did not show significant enhancement of the downregulation of the MDM2 mRNA levels by cyclosporine A (CsA, a calcineurin inhibitor) (Figure 3D). Furthermore, enforced NFAT1 expression increased the MDM2 luciferase activity $(P<0.001)$, which was reduced by JapA treatment $(P<0.05)$ (Figure 3E). Similar results were observed when NFAT1 was activated using ION (Figure 3F).

\section{JapA inhibits the nuclear localization of NFAT1}

After translocation into the nucleus, NFAT1 transactivates its downstream target genes, e.g., c-Myc, 

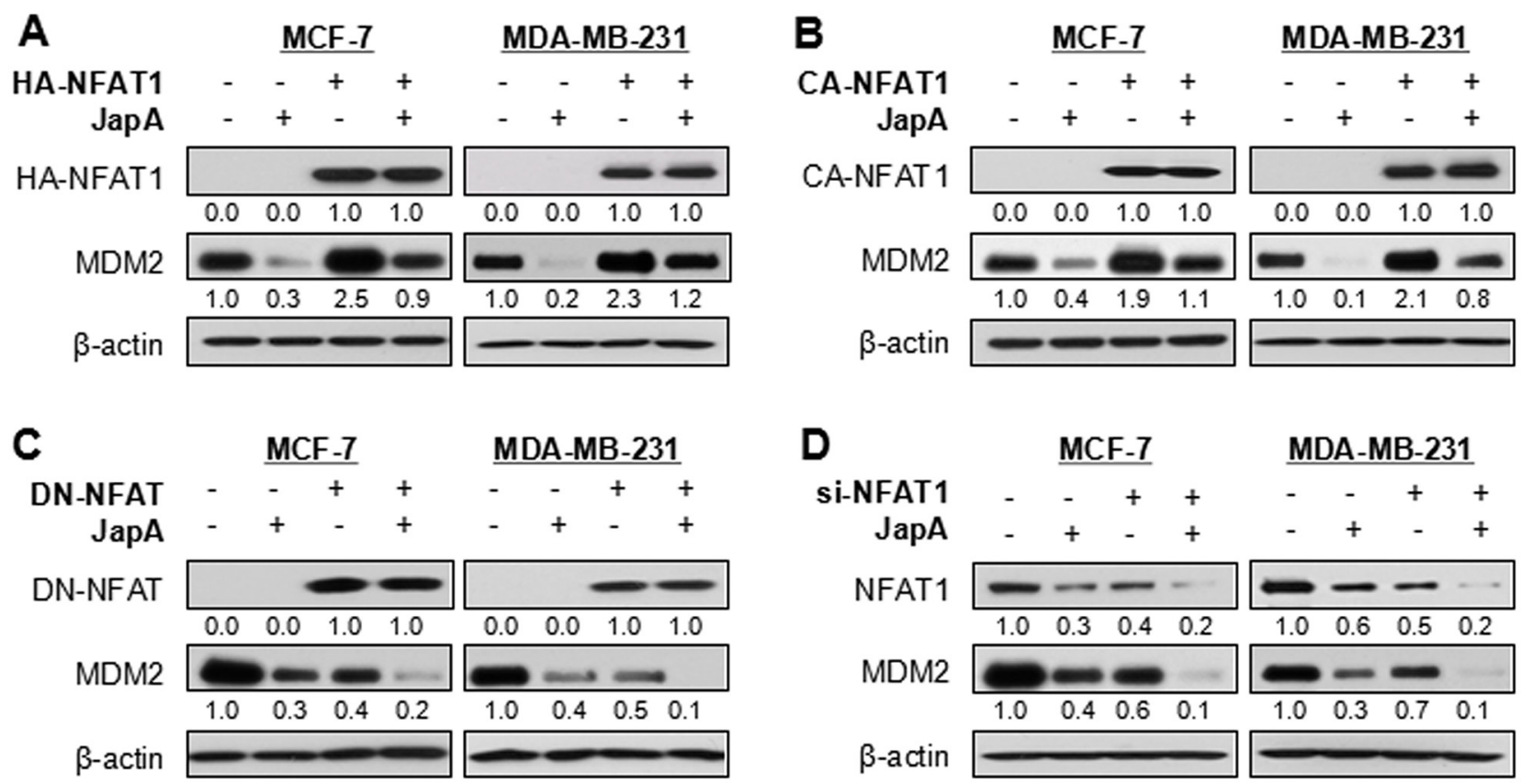

Figure 2: Effects of JapA on NFAT1-mediated MDM2 protein expression. A, B. MCF-7 and MDA-MB-231 cells were transfected with (A) a HA-tagged NFAT1 plasmid or (B) a HA-tagged constitutively activated NFAT1 (CA-NFAT1) plasmid, followed by treatment with JapA (1 and $2 \mu \mathrm{M}$, respectively, for MCF-7 and MDA-MB-231 cells) for $24 \mathrm{~h}$. The NFAT1 protein levels were detected by Western blotting using antibody against HA. C, D. The cells were transfected with a FLAG-tagged dominant-negative NFAT (DN-NFAT) plasmid, or with NFAT1 siRNA (si-NFAT1), followed by treatment with JapA for $24 \mathrm{~h}$. The NFAT1 protein levels were detected by Western blotting using antibodies against FLAG (C) or NFAT1 (D), respectively. The intensity ratio under each band was obtained by IMAGEJ software analysis normalized on untreated control. All of the experiments were repeated three times.

COX-2, and MDM2, resulting in cancer cell proliferation and migration [31-34]. Therefore, we further examined the effects of JapA on NFAT1 localization in both MCF-7 and MDA-MB-231 cell lines. As shown in Figure 4A, JapA decreased the protein levels of both cytoplasmic and nuclear NFAT1, which could be partially restored by ION and further decreased by CsA. The inhibitory effects of JapA on NFAT1 nuclear localization were also confirmed by an immunofluorescence study in both cell lines. As shown in Figure 4B, JapA treatment decreased the immunostaining of NFAT1 in the nucleus, which led to the repression of its transcriptional activity.

\section{JapA inhibits the interaction between NFAT1 and the MDM2 $\mathrm{P} 2$ promoter}

Since NFAT1 activates $M D M 2$ transcription through direct binding to the MDM2 $\mathrm{P} 2$ promoter [31], we then examined whether JapA affects the interaction between NFAT1 and the MDM2 P2 promoter. As shown in Figure 5A, an electrophoretic mobility shift assay (EMSA) showed that endogenous NFAT1 from MDA-MB-231 cells was specifically bound to the MDM2 P2 promoter, and this binding was significantly reduced by JapA treatment. In addition, the nuclear extracts from ION-treated cells showed the enhanced binding of NFAT1 to the MDM2 probe, whereas simultaneous treatment with CsA inhibited the binding (Figure 5A). We then performed a chromatin immunoprecipitation (ChIP) assay combined with a realtime quantitative PCR analysis. As shown in Figure 5B, endogenous NFAT1 from MDA-MB-231 cells specifically bound the P2 promoter of MDM2 $(P<0.01)$, and JapA reduced the binding of NFAT1 to the MDM2 $\mathrm{P} 2$ promoter $(P<0.05)$.

\section{JapA induces the ubiquitination and proteasomal degradation of NFAT1}

We next examined the mechanisms responsible for the JapA-induced NFAT1 inhibition. As shown in Figure 6A, JapA increased NFAT1 protein degradation in the presence of a protein synthesis inhibitor, cycloheximide (CHX), shortening the half-life of the NFAT1 protein in both MCF-7 and MDA-MB-231 cell lines. Further studies showed that the NFAT1 reduction induced by JapA was prevented by MG-132 (a proteasome inhibitor) treatment (Figure 6B), which indicated that JapA could induce the proteasomal degradation of NFAT1. These results were supported by the fact that JapA promoted NFAT1 ubiquitination (Figure 6C). Together, JapA promoted the ubiquitination and proteasomal degradation of NFAT1, and thereby inhibited the expression and activity of NFAT1 in breast cancer cells in vitro and in vivo. 

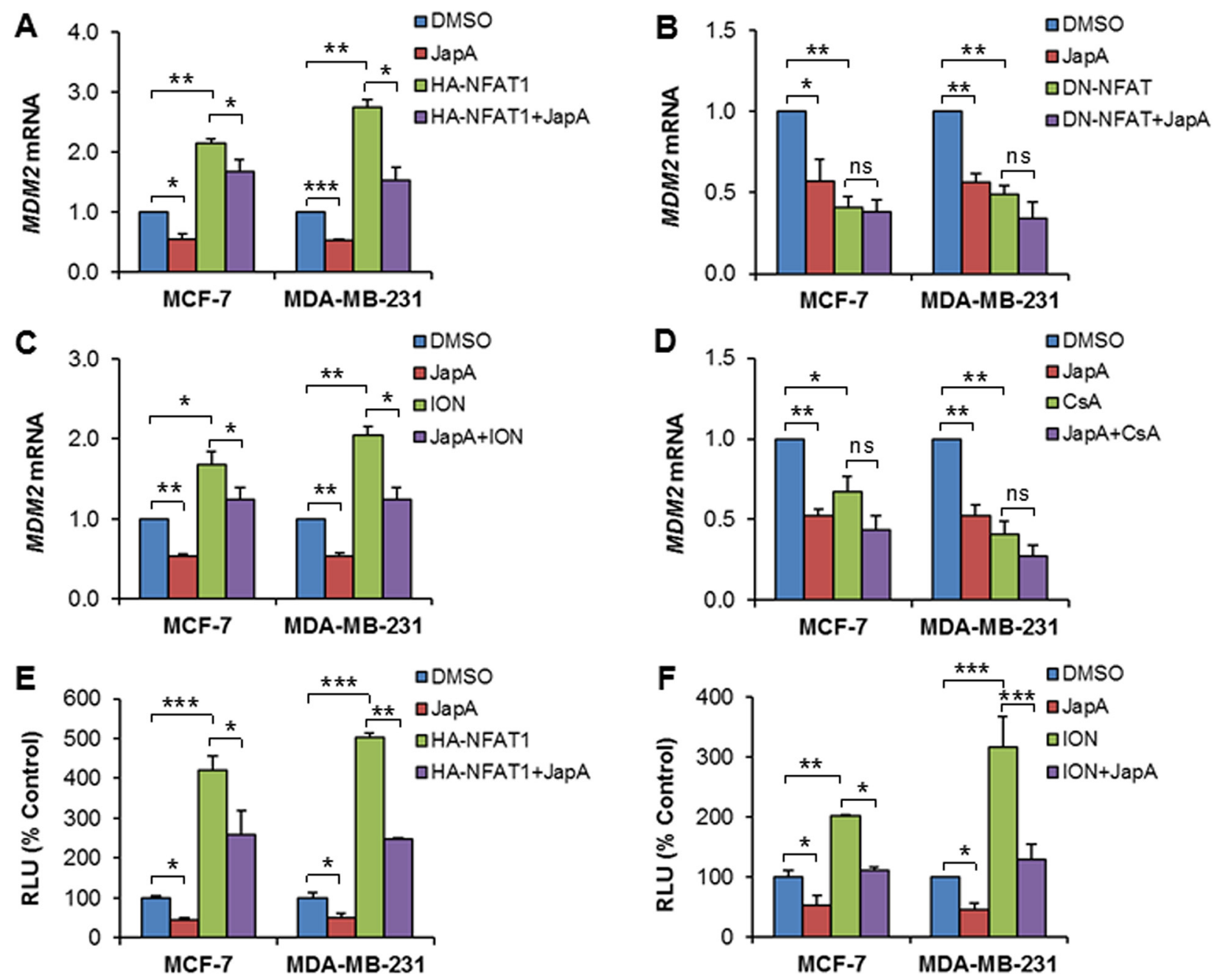

Figure 3: Effects of JapA on NFAT1-mediated $\boldsymbol{M D M} 2$ transcription. A, B. MCF-7 and MDA-MB-231 cells were transfected with (A) an HA-tagged NFAT1 plasmid or (B) a FLAG-tagged dominant-negative NFAT (DN-NFAT) plasmid, followed by treatment with JapA ( 1 and $2 \mu \mathrm{M}$, respectively, for MCF-7 and MDA-MB-231 cells) for $24 \mathrm{~h}$. The relative mRNA levels of $M D M 2$ were determined by real-time quantitative PCR. C, D. The cells were treated with JapA in the presence or absence of (C) ionomycin (ION; $4 \mu \mathrm{M})$ or (D) cyclosporine A $(\mathrm{CsA} ; 2 \mu \mathrm{M})$ for $24 \mathrm{~h}$. The relative mRNA levels of $M D M 2$ were determined by real-time quantitative PCR. E, F. The cells were transfected with a HA-NFAT1 plasmid combined with the full-length (Luc 01) MDM2 P2 promoter (E), or with the full-length (Luc 01) MDM2 P2 promoter alone (F), followed by treatment with JapA (E), or JapA with or without ION (4 $\mu$ M) (F) for 24 h. The MDM2 luciferase activity was determined using the Dual-Luciferase Reporter Assay System. All of the experiments were repeated three times. $(* P<0.05, * * P<0.01$, and $* * * P<0.001$, "ns" denotes "not significant").

\section{The cell response to JapA is affected by NFAT1 overexpression and knockdown in breast cancer cells}

The importance of NFAT1 and NFAT1-MDM2 pathway in JapA's anti-breast cancer activity was further demonstrated using Tet-on inducible NFAT1 overexpression (OE) and siRNA NFAT1 knockdown (KD) MCF-7 cells. Tet-induced NFAT1 OE upregulated the MDM2 expression (Figure 7A), and reduced the effects of JapA on NFAT1-MDM2 signaling (Figure 7A), colony formation (Figure 7B), and apoptosis (Figure 7C) in MCF-7 cells. In contrast, NFAT1 KD decreased the MDM2 expression levels (Figure 7D), and enhanced the effects of JapA on NFAT1-MDM2 pathway (Figure 7D), colony formation (Figure 7E), and apoptosis (Figure 7F) in MCF-7 cells.

We further demonstrated the importance of NFAT1 and NFAT1-MDM2 pathway in JapA's anticancer activity using NFAT1 OE and KD MDA-MB-231 (p53 mutant) cells. NFAT1 OE in MDA-MB-231 cells activated MDM2 (Figure 8A) and reduced the effects of JapA on NFAT1MDM2 pathway (Figure 8A), cell viability (Figure 8B), and apoptosis (Figure 8C). However, NFAT1 KD decreased the expression levels of MDM2 (Figure 8D) and enhanced the effects of JapA on NFAT1-MDM2 pathway (Figure 8D), cell viability (Figure 8E), and apoptosis (Figure 8F) in MDA-MB-231 cells. 
A

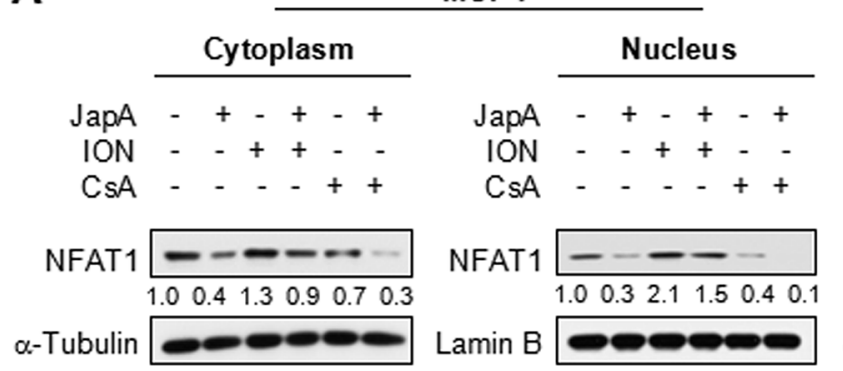

B
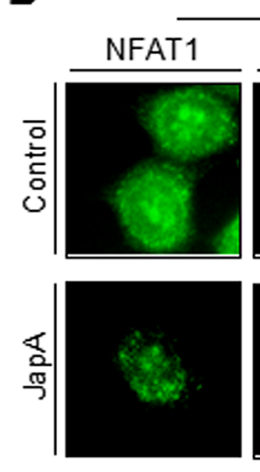

MCF-7

7

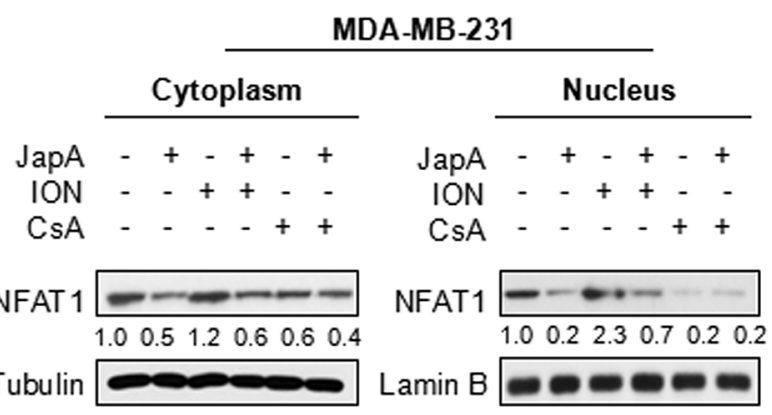

MDA-MB-231

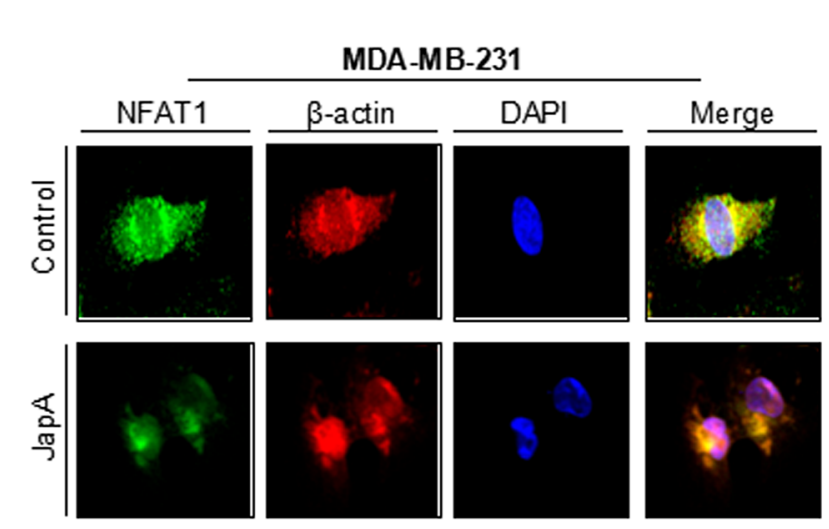

Figure 4: Effects of JapA on the nuclear localization of NFAT1. A. MCF-7 and MDA-MB-231 cells were treated with JapA (1 and $2 \mu \mathrm{M}$, respectively) in the presence or absence of ionomycin (ION; $4 \mu \mathrm{M}$ ) or cyclosporine A (CsA; $2 \mu \mathrm{M})$ for $24 \mathrm{~h}$. The nuclear and cytosolic proteins were extracted and examined by Western blotting. Lamin B and $\alpha$-tubulin were used as the internal references, respectively. The intensity ratio under each band was obtained by IMAGEJ software analysis normalized on untreated control. B. The cells were treated with JapA or vehicle for $12 \mathrm{~h}$, followed by immunofluorescence detection. $\beta$-actin and DAPI were used as internal references. All of the experiments were repeated three times.

\section{DISCUSSION}

In the present study, we demonstrated that the newly discovered MDM2 inhibitor JapA inhibits NFAT1 and NFAT1-MDM2 signaling, which plays a critical role in its anticancer activity. Our findings provide evidence for the value of NFAT1 and NFAT1-MDM2 signaling as novel targets in developing effective targeted therapy for human cancers.

We have made several important discoveries in the present study. First, JapA represents a new class of NFAT1 inhibitors that target both NFAT1 and NFAT1MDM2 signaling. Second, JapA inhibits the expression and activity of NFAT1 in breast cancer cells in vitro and in vivo. Third, JapA inhibits NFAT1-mediated expression of the transcriptional responsive genes by inhibiting NFAT1 nuclear localization and disrupting NFAT1-DNA binding. Fourth, JapA induces NFAT1 protein ubiquitination and proteasomal degradation. Finally, the inhibition of NFAT1 and NFAT1-MDM2 pathway is critical for JapA's anticancer activity. Our results demonstrated the characterization of JapA as a novel NFAT1 inhibitor and the importance of NFAT1 and NFAT1-MDM2 signaling in JapA's inhibitory effect against human cancer.

Our previous studies have demonstrated that JapA is a selective and potent inhibitor of MDM2 and has novel molecular mechanisms distinct from other MDM2 inhibitors under development [13]. JapA directly binds to MDM2 protein and induces MDM2 autoubiquitination and proteasomal degradation. JapA also inhibits $M D M 2$ transcription in an NFAT-dependent manner, suggesting that JapA inhibits MDM2 via alternative mechanisms different from the MDM2 inhibitors that target MDM2-p53 interaction and MDM2 itself. It has been reported that the transcription factor NFAT1 activates MDM2, independent of p53 [31]. In the present study, we demonstrated that the inhibitory effects of JapA on $M D M 2$ transcription depend on the inhibition of NFAT1, regardless of the p53 status of the cells.

NFAT proteins (NFAT1-NFAT5) are a group of inducible transcription factors and have been implicated in many biological processes, including cancer development and progression [24-26]. We and other investigators have suggested NFAT as a potential molecular target for cancer therapy [24-26]. NFAT1, also named NFATp or NFATc2, is overexpressed and hyperactivated in human cancers, including breast cancer [27-30]. Aberrant activation of NFAT1 signaling results in the upregulation of genes associated with tumor growth and metastasis, such as MDM2 and c-Myc [24-26]. Recent evidences have suggested that NFAT1 plays a critical role in breast cancer development and progression. It has been found that 

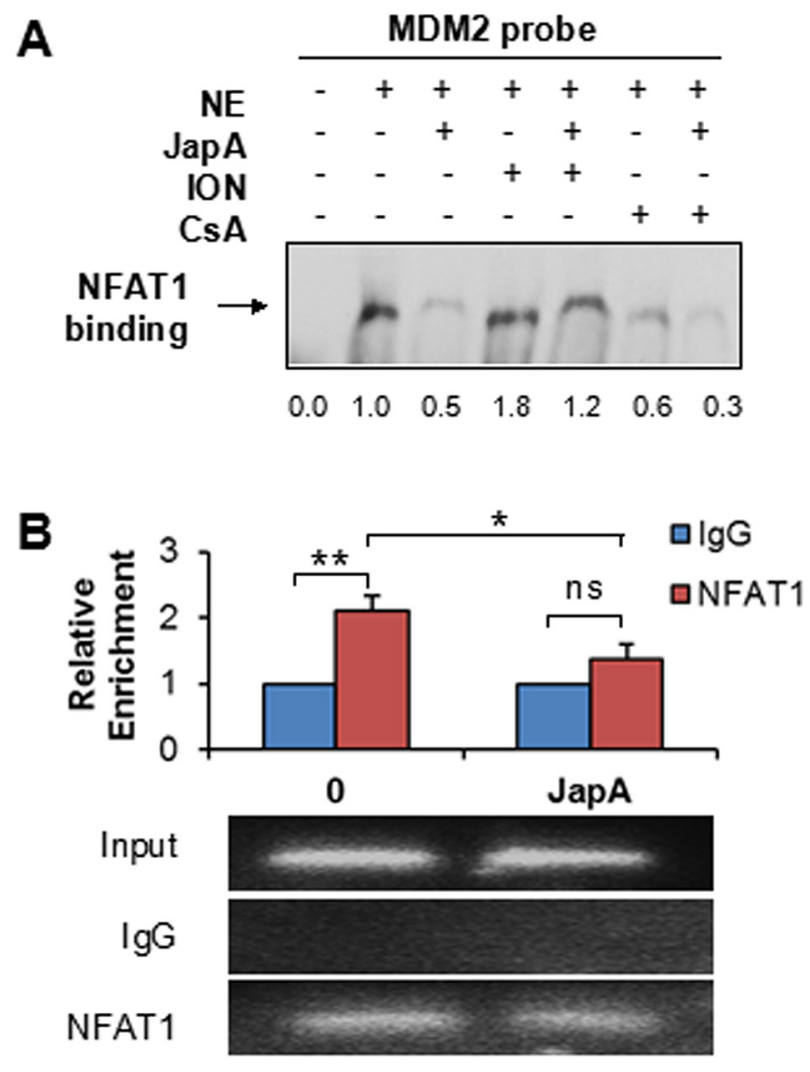

Figure 5: Effects of JapA on the binding of NFAT1 to the MDM2 P2 promoter. A. Nuclear extracts from MDA-MB-231 cells treated with JapA $(2 \mu \mathrm{M})$ in the presence or absence of ionomycin (ION; $4 \mu \mathrm{M})$ or cyclosporine A $(\mathrm{CsA} ; 2 \mu \mathrm{M})$ were incubated with the MDM2 probe. An EMSA assay was performed to detect the binding between endogenous NFAT1 protein and the MDM2 probe. The intensity ratio under each band was obtained by IMAGEJ software analysis normalized on untreated control. B. MDA-MB-231 cells were treated with JapA $(2 \mu \mathrm{M})$ or the vehicle for $24 \mathrm{~h}$. The cell lysates were immunoprecipitated with NFAT1 or IgG antibodies. The DNA bound to the endogenous NFAT1 was eluted and quantified using primers specific for the MDM2 P2 promoter by PCR. All of the experiments were repeated three times. ( $* P<0.05$ and $* * P<0.01$, "ns" denotes "not significant").

NFAT1 promotes breast cancer cell motility and invasion by regulating COX-2 and glypican-6 [30, 34-35]. NFAT1 also functions as a critical factor in Akt/protein kinase B (PKB) and Glycogen synthase kinase $3 \beta$ (GSK3 $\beta$ ) signaling pathways to regulate the breast cancer metastasis [36]. In addition, NFAT1 promotes intratumoral neutrophil infiltration by regulating interleukin-8 (IL8) expression in breast cancer [37]. NFAT1 and signal transducer and activator of transcription 5 (Stat5) signaling cascades have been found to antagonize each other in breast cancer, which may affect breast cancer initiation, growth, and metastasis [28]. Importantly, the constitutive activation of NFAT1 signaling has been observed in diagnostic breast cancer cases, and it is essential to the tumorigenic and metastatic potential of breast cancer cells [38]. Therefore, targeting NFAT1 is a promising strategy for developing novel and effective anti-breast cancer therapy.

NFAT1 exerts its oncogenic functions in human cancer through calcineurin-dependent and -independent pathways [24-26]. Once activated, calcineurin directly dephosphorylates NFAT1 protein, leading to its nuclear translocation and the transactivation of its target genes
$[24,26]$. The balance between nuclear import and export of the NFAT1 protein is also regulated by various kinases, such as GSK3 $\beta$, casein kinase 1 (CK1), and protein kinase A (PKA), independent of calcineurin [24]. Several strategies have been demonstrated to develop novel NFAT inhibitors. First, targeting the upstream regulators of NFAT proteins, such as calcineurin and GSK3 $\beta$, to inhibit NFAT protein dephosphorylation and nuclear translocation; second, directly targeting NFAT proteins themselves to inhibit NFAT expression and nuclear translocation; and third, blocking NFAT-DNA binding to inhibit NFAT transcriptional activity. Thus far, most of the NFAT inhibitors under development have been designed by following the first strategy, such as VIVIT peptides [39-40], PxIxIT peptides [41], CsA [42-44], FK-506 [44-45], and NCI3 [46].

Increasing evidences have suggested that different NFAT isoforms even have opposite roles in the same cancer type. For example, the overexpression and hyperactivation of NFAT1 and NFAT2 are frequently observed in human cancer and contribute to cancer development, progression, and metastasis [24, 26, 31, 47-49]. 

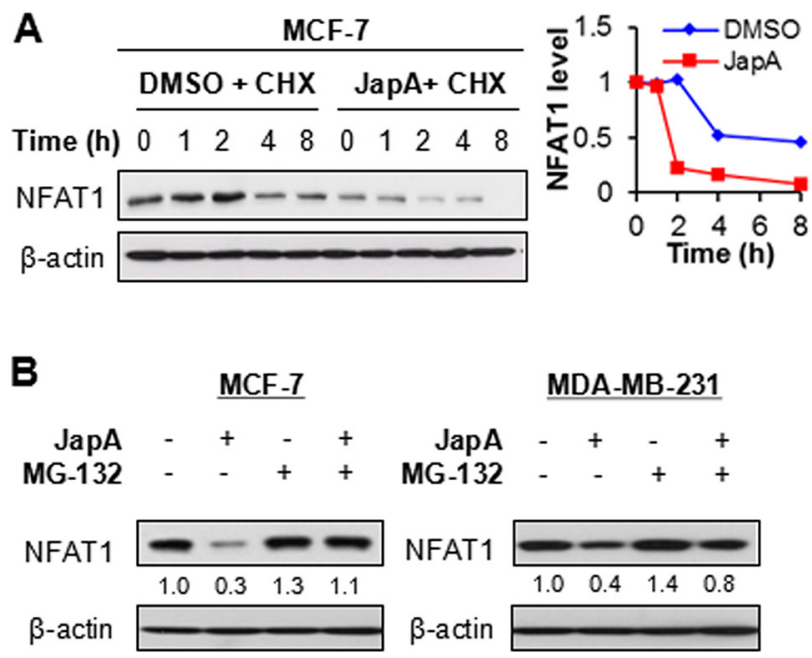
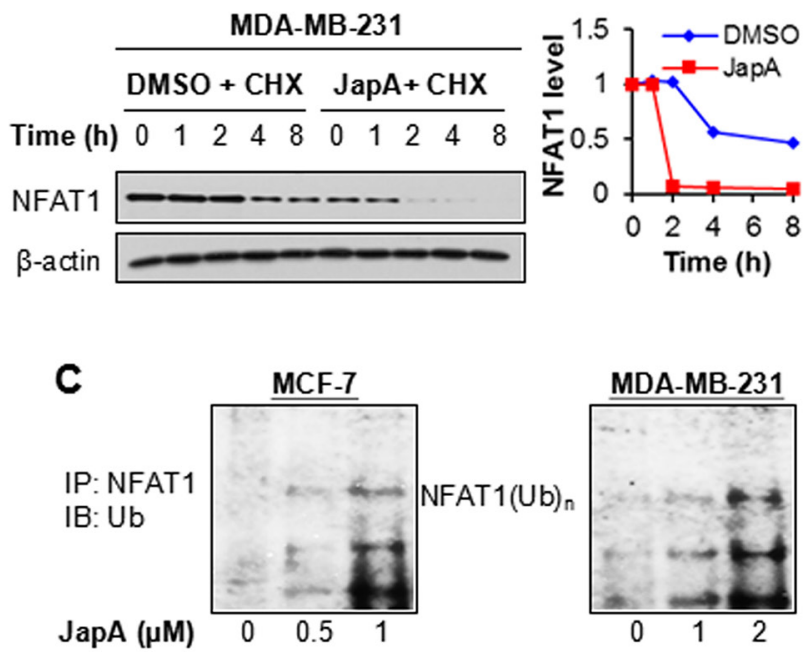

Figure 6: Effects of JapA on NFAT1 protein stability. A. MCF-7 and MDA-MB-231 cells were treated with JapA or vehicle, followed by exposure to a protein synthesis inhibitor, cycloheximide (CHX, $15 \mu \mathrm{g} / \mathrm{mL}$ ). The NFAT1 protein levels were detected by Western blotting at the indicated times after exposure to CHX. Graphs (right) show the quantification of the immunoblotting data. B. The cells were treated with JapA or vehicle ( 1 and $2 \mu \mathrm{M}$, respectively, for MCF-7 and MDA-MB-231 cells) for $24 \mathrm{~h}$, then exposed to MG-132 $(25 \mu \mathrm{M})$, a proteasome inhibitor, for an additional $6 \mathrm{~h}$. The protein levels of NFAT1 were detected by Western blotting. The intensity ratio under each band was obtained by IMAGEJ software analysis normalized on untreated control. C. The cells were co-transfected with NFAT1 and ubiquitin plasmids, followed by treatment with JapA for $24 \mathrm{~h}$. Cell lysates were subjected to immunoprecipitation with an NFAT1 antibody. The ubiquitinated NFAT1 was detected using an anti-ubiquitin antibody. All of the experiments were repeated three times.

However, NFAT3 has been reported to be specifically expressed in estrogen receptor $\alpha$ positive breast cancer cells and reduce cancer cell motility by inhibiting the expression of Lipocalin 2 [50]. Up to date, most of the NFAT inhibitors have been developed to nonspecifically inhibit all calcineurin-responsive NFAT isoforms no matter whether they exert their roles as an oncogene or a tumor suppressor, resulting in suboptimal efficacy against human cancer and unexpected side effects on human immune system. Several NFAT inhibitors that target either oncogenic NFAT isoforms, such as helenalin [51] and zoledronic acid [52], or NFAT-DNA binding, such as imperatorin [53] and digitoxin [54] have shown significant anticancer activities in vitro and in vivo. The present study reports, for the first time, a novel NFAT1 inhibitor JapA that targets both oncogenic NFAT1 and NFAT1 interaction with the $M D M 2 \mathrm{P} 2$ promoter.

We have demonstrated that JapA is a novel NFAT1 inhibitor. Several assays have been performed to demonstrate the specificity of JapA in targeting NFAT1 and NFAT1-MDM2 pathway in breast cancer cells. First, the inhibitory effects of JapA on the expression of NFAT1 and its target genes, MDM2, c-Myc and COX-2, were shown in human breast cancer cells in vitro and breast xenograft tumors in vivo. Second, the inhibitory effects of JapA on NFAT1-mediated MDM2 activation were demonstrated using breast cancer cells with NFAT1 OE or KD, as well as other pharmacological inhibitors and activators of calcineurin-NFAT signaling. Third, the inhibitory effects of JapA on NFAT1 nuclear localization and NFAT1DNA binding were demonstrated using immunostaining,
EMSA and CHIP assays. NFAT1 activates MDM2 through interacting with the MDM2 $\mathrm{P} 2$ promoter but not the P1 promoter, while our results have shown that JapA inhibits the binding of NFAT1 to MDM2 P2 promoter, independent of p53. Importantly, the positive regulation of MDM2 by NFAT1 has been observed in human hepatocellular carcinoma tissue samples, regardless of the p53 status of the tumors [13]. Since JapA represents a new class of MDM2 inhibitors that inhibit both MDM2 and its activator NFAT1, it could be more effective than other NFAT1 or MDM2 inhibitors in treating tumors with high expression levels of both NFAT1 and MDM2. Moreover, its excellent efficacy for treating tumors with both wild-type p53 and non-functional p53 is also expected. Fourth, the JapAinduced NFAT1 protein destabilization was attributed to NFAT1 ubiquitination and proteasomal degradation, which was elucidated using protein synthesis inhibitor and proteasome inhibitor and determined by an ubiquitination assay. Although it has been reported that the NFAT1 ubiquitination is mediated by MDM2 through its E3 ubiquitin ligase activity [30], more direct evidence is still needed for the role of MDM2 in JapA-induced NFAT1 protein degradation.

We have further demonstrated that NFAT1 and NFAT1-MDM2 pathway play a critical role in JapA's inhibitory effects against human cancer, as indicated in NFAT1 OE and KD MCF-7 (p53 wild-type) and MDA-MB-231 (p53 mutant) breast cancer cell lines. Despite these results, there is no direct evidence that JapA just specifically inhibits NFAT1 and further studies on its effects on other NFAT isoforms are needed. The 
A

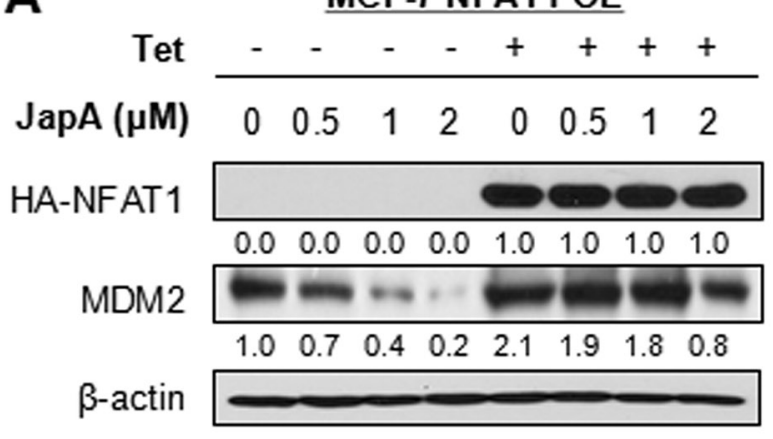

B

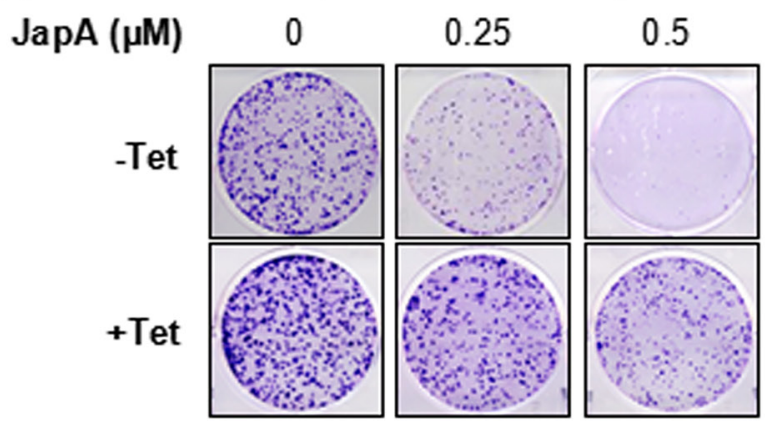

C

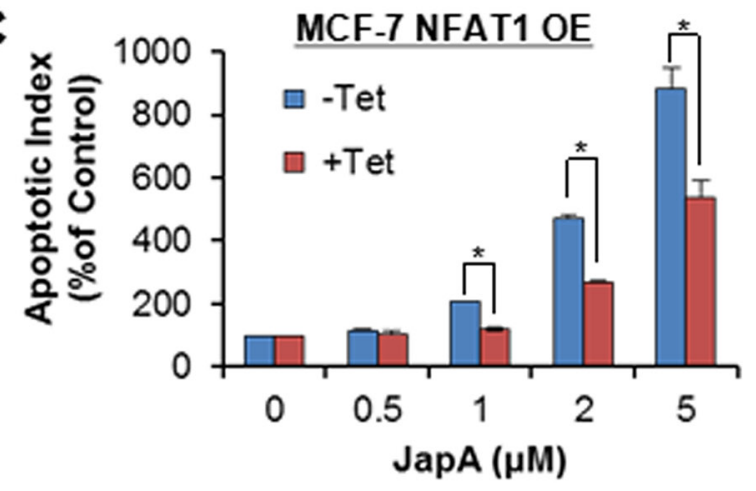

D

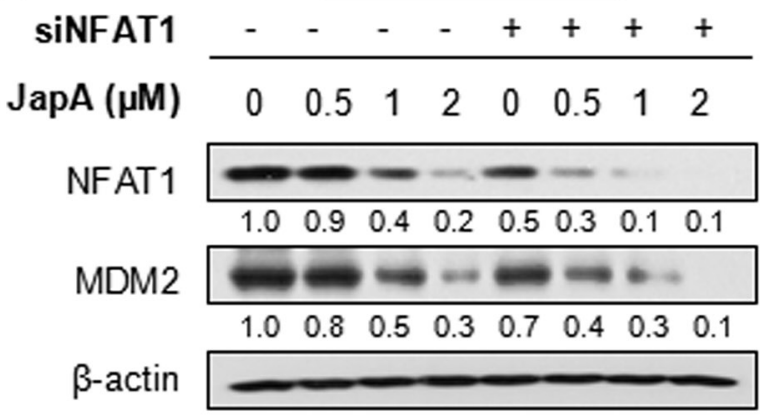

$\mathbf{E}$

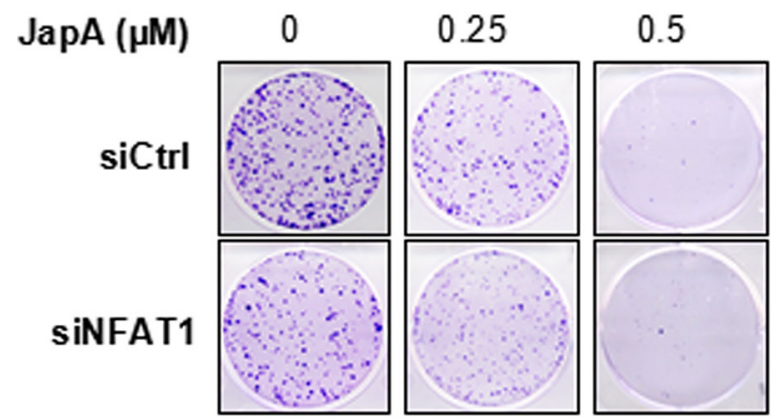

$\mathbf{F}$

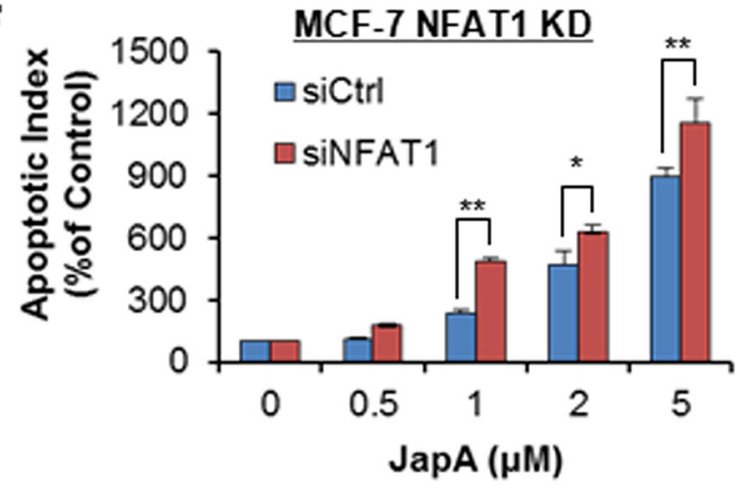

Figure 7: NFAT1 overexpression and knockdown affect the cell response to JapA in breast cancer cells. A, B. and C. The inducible NFAT1 overexpression MCF-7 cells were incubated with (+Tet; $1 \mu \mathrm{g} / \mathrm{mL}$ ) or without tetracycline (-Tet) for $24 \mathrm{~h}$ and then treated by various concentrations of JapA for (A) $24 \mathrm{~h}$ for the expression of NFAT1 and MDM2; (B) $24 \mathrm{~h}$ for the colony formation assay; and (C) $48 \mathrm{~h}$ for the apoptosis assay. D, E. and F. MCF-7 cells were transfected with control siRNA or a NFAT1 siRNA for $36 \mathrm{~h}$, followed by treatment with various concentrations of JapA for (D) $24 \mathrm{~h}$ for the expression of NFAT1 and MDM2; (E) $24 \mathrm{~h}$ for the colony formation assay; and (F) $48 \mathrm{~h}$ for the apoptosis assay. The intensity ratio under each band was obtained by IMAGEJ software analysis normalized on untreated control. All assays were performed in triplicate and repeated three times $\left(* P<0.05\right.$ and $\left.{ }^{* *} P<0.01\right)$.

determination and validation of the direct binding of JapA to NFAT1 would help address these issues partially. Moreover, it is possible that JapA may have other molecular targets that are important for its anticancer activity; further exploration of additional targets also needs to be considered, such as MDMX and other oncogenic NFAT isoforms.

Of note, we have shown the in vivo efficacy of JapA and its safety profile at two dose levels $(15$ and $30 \mathrm{mg} /$ $\mathrm{kg} /$ day) in breast cancer xenograft models [13]. We have found that JapA effectively inhibits the tumor growth and the protein expression of MDM2 and NFAT1 in vivo, without inducing any apparent host toxicity. It is expected that JapA at these dose levels is safe and effective in treating other tumors with high expression levels of MDM2 and NFAT1, regardless of p53 status of the tumors. However, the toxicological profile of JapA needs to be further characterized using more clinically relevant animal 

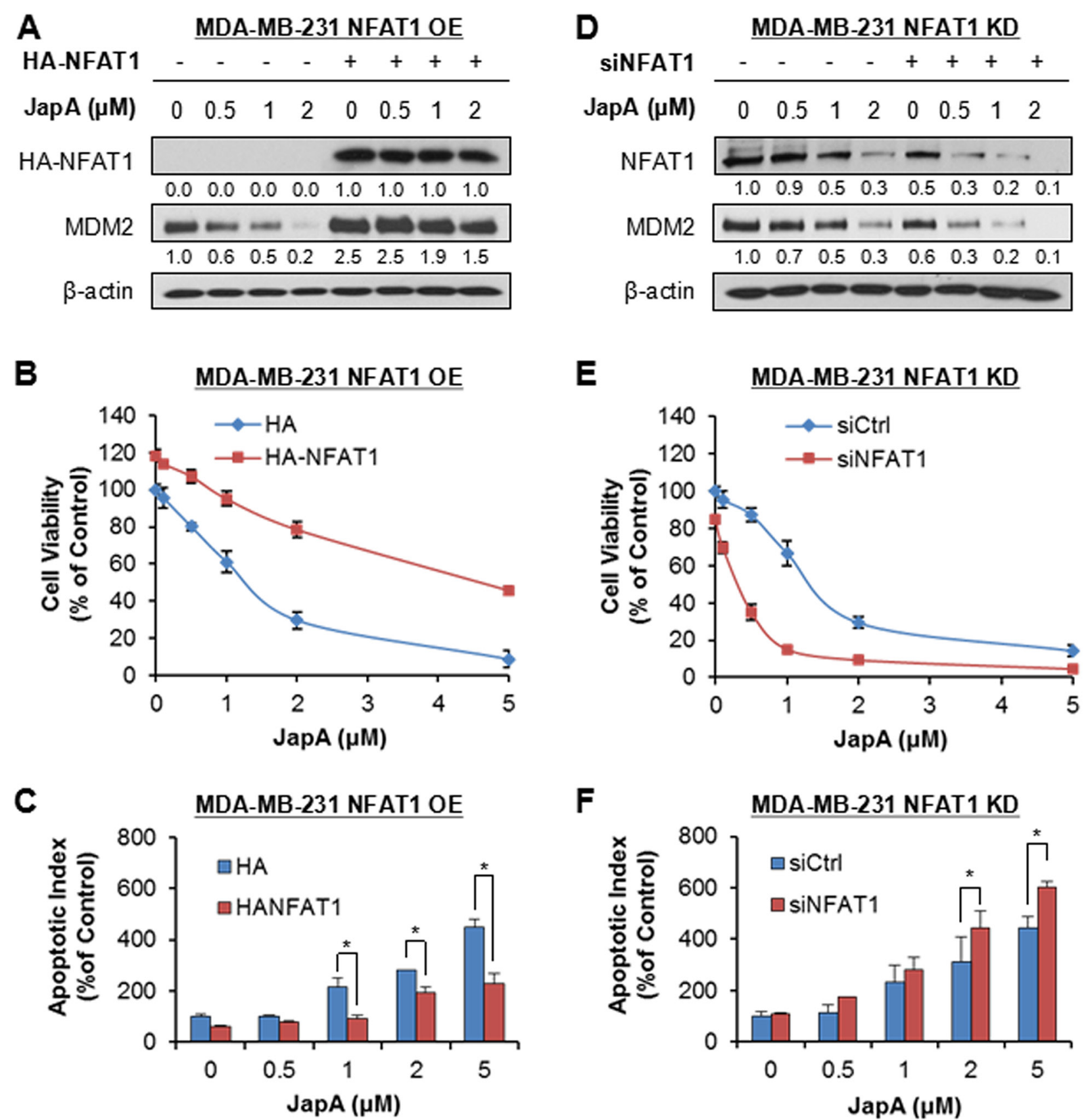

Figure 8: NFAT1 overexpression and knockdown affect the cell response to JapA in breast cancer cells. A, B. and C. MDA-MB-231 cells were transfected with an HA plasmid or an HA-tagged NFAT1 plasmid for $24 \mathrm{~h}$ and then treated with various concentrations of JapA for (A) $24 \mathrm{~h}$ for the expression levels of HA-NFAT1 and MDM2; (B) $72 \mathrm{~h}$ for determination of the cell viability; and (C) $48 \mathrm{~h}$ for the apoptosis assay. D, E. and F. MDA-MB-231 cells were transfected with NFAT1 siRNA or the respective control siRNA for $36 \mathrm{~h}$ and then treated with various concentrations of JapA for (D) $24 \mathrm{~h}$ for expression levels of NFAT1 and MDM2; (E) $72 \mathrm{~h}$ for determination of the cell viability; and (F) $48 \mathrm{~h}$ for the apoptosis assay. The intensity ratio under each band was obtained by IMAGEJ software analysis normalized on untreated control. All assays were performed in triplicate and repeated three times $(* P<0.05)$.

models, especially syngeneic models, since NFAT1 plays a critical role in human immune system and developmental programs. The effects of JapA on the expression and activity of NFAT1 should be further examined in both immune cells and tumor cells to determine if JapA affects the immune system or it is selective for tumor cells.
In conclusion, our study demonstrates that JapA inhibits the expression and activity of oncogenic NFAT1 through inhibiting NFAT1 nuclear localization and NFAT1DNA binding and promoting NFAT1 ubiquitination and proteasomal degradation, which contributes to the anticancer activity of JapA. These results indicate that JapA 
is a novel anticancer drug candidate with a dual-targeting mechanism and provide new insights into the future development of novel dual inhibitors of NFAT1 and MDM2 for cancer therapy. However, it is tempting to speculate that the unique inhibitory effects of JapA on NFAT1-MDM2 pathway and its efficacy and toxicological profile should be further explored for the elucidation of uncharacterized mechanisms of action, as well as the development of safe and effective anticancer therapy.

\section{MATERIALS AND METHODS}

\section{Cell culture}

Human breast cancer (MCF-7, MDA-MB-231, and MDA-MB-468) and non-malignant epithelial (MCF-10A) cells were obtained from the American Type Culture Collection (Rockville, MD). Human mammary luminal epithelial (HMLE) cells were obtained from Zen-Bio, Inc. (Research Triangle Park, NC). The MCF-7 p53 ${ }^{--}$and the inducible NFAT1 OE MCF-7 cell lines were established previously [31, 55-56]. HMLE cells were grown in mammary luminal epithelial cell growth medium (Zen-Bio, Inc.). Other cell lines were cultured as reported by us earlier [13].

\section{Chemicals, reagents, antibodies, plasmids, and siRNA}

JapA was obtained as previously reported [13], with the purity being $>95 \%$ as confirmed by IR, ESI-MS, NMR, and HPLC/MS ${ }^{\mathrm{n}}$ [16-17]. All chemicals and solvents were of the highest analytical grade available. The anti-human NFAT1 (1/NFAT-1) antibody was from BD Biosciences (San Jose, CA). The anti-human COX-2 (C-20) and c-Myc (0.N.222) antibodies were from Santa Cruz Biotechnology Inc. (Dallas, TX). The anti-human MDM2 (Ab-2) antibody was from Calbiochem (Billerica, MA). The anti-human ubiquitin (6C1) and $\beta$-actin (AC-15) antibodies were from Sigma (St. Louis, MO). Goat anti-mouse $\operatorname{IgG}(\mathrm{H}+\mathrm{L})$ and goat anti-rabbit IgG $(\mathrm{H}+\mathrm{L})$ were obtained from Bio-Rad (Hercules, CA). The human full-length $M D M 2$ P2 promoter reporters were kind gifts from Dr. J.P. Blaydes (Southampton General Hospital, UK). Vectors expressing HA-NFAT1, CA-NFAT1, and DN-NFAT were kindly provided by Dr. C.W. Chow (Yeshiva University). NFAT1 siRNA or control siRNA were from Thermo Scientific (Rockford, IL). Both plasmids and siRNAs were transfected into cells using the same protocols as we reported previously [57].

\section{Assays for cell viability, colony formation and apoptosis}

The cells were treated with various concentrations of JapA, and the cell viability, cell colony formation and apoptosis assays were performed as described previously [58-59].

\section{Real-time quantitative PCR, immunoblotting, and luciferase assay}

For real-time quantitative PCR analysis, total RNA was extracted using the Trizol reagent (Invitrogen, Grand Island, NY) and analyzed as described previously [59-60]. For immunoblotting assay, cell lysates and tissue homogenates were prepared in NP40 lysis buffer containing protease inhibitors (Sigma, St Louis, MO). The protein concentration was estimated using the Bradford reagent (Bio-Rad, Hercules, CA). The expression levels of various proteins were examined as described previously [58-59]. For luciferase assay, cells were co-transfected with full-length human MDM2 P2 promoter vector with the Renilla luciferase reporter as an internal control [13]. After exposure to JapA for $24 \mathrm{~h}$, the luciferase activity of the MDM2 promoter reporters was determined using the Dual-Luciferase Reporter Assay System (Promega, Madison, WI).

\section{Ubiquitination assay}

Cells were co-transfected with NFAT1 and ubiquitin plasmids and treated with various concentrations of JapA. Cell lysates were immunoprecipitated with antiNFAT1 antibody, and the bound proteins were purified with protein G-Sepharose beads (Sigma, St Louis, MO), resolved on SDS-PAGE, and detected by an anti-ubiquitin antibody [13].

\section{Electrophoretic mobility shift assay (EMSA)}

MDA-MB-231 cells were treated with JapA $(2 \mu \mathrm{M})$, ION $(4 \mu \mathrm{M})$, and/or CsA $(2 \mu \mathrm{M})$ for $24 \mathrm{~h}$, and nuclear factions were extracted using the NE-PER Nuclear and Cytoplasmic Extraction Kit (Thermo Scientific, Rockford, IL). The NFAT1 DNA-binding activity was measured as described previously [31]. The prepared nuclear extracts were preincubated with $1 \mu \mathrm{g}$ of poly-(dI:dC) (Thermo Scientific, Rockford, IL) and then reacted with a biotin-labeled MDM2 probe at room temperature for $30 \mathrm{~min}$. The sequences of the biotin-labeled probe were MDM2 forward, 5'-gcaggttgactcagcttttcctcttgagctggtcaagttca-3' and MDM2 reverse, 5'-tgaacttgaccagctcaagaggaaaagctgagtcaacctgc-3'.

\section{Chromatin immunoprecipitation (ChIP) assay}

MDA-MB-231 cells were treated with DMSO or JapA $(2 \mu \mathrm{M})$ for $24 \mathrm{~h}$, then the chromatin immunoprecipitation (ChIP) assay was performed in the treated cells as described previously [31]. The specific primer pairs used were: 5'-ccccegtgacctttaccetg-3' and $5^{\prime}$-agcctttgtgcggttcgtg-3' for qualitative or quantitative PCR amplification of the responsive element of the MDM2 promoter. 


\section{Cell immunofluorescence assay and immunohistochemistry}

For immunofluorescence assay, cells were seeded on coverslips in a 12-well plate at a density of 10,000 cells/ well, allowed to attach overnight, and treated with JapA for $12 \mathrm{~h}$. The cells were then processed for immunofluorescence detection as reported [58]. For the immunohistochemical studies, the fresh tumor tissues were dissected from the mice bearing both MCF-7 and MDA-MB-231 xenograft tumors [13]. These breast cancer xenograft models were developed by following the animal study protocols that have been approved by the Institutional Animal Use and Care Committee of the Texas Tech University Health Sciences Center. The dose, dose frequency, and administration route were chosen based on the previous studies $[13,18,20]$. The dissected tumor tissues were fixed and stained for the protein expression and location of NFAT1 following the reported protocols [13].

\section{Statistical analysis}

The data are expressed as the means \pm SEM from at least three independent experiments. Two-sided $t$-test was used for comparisons between two groups. $P<0.05$ was considered as statistically significant.

\section{Abbreviations}

CA-NFAT1, constitutively-activated NFAT1; ChIP, chromatin immunoprecipitation; CHX, cycloheximide; CK1, casein kinase 1; COX-2, cyclooxygenase 2; CsA, cyclosporine A; DN-NFAT, dominant-negative NFAT; EMSA, electrophoretic mobility shift assay; GSK $3 \beta$, glycogen synthase kinase $3 \beta$; HMLE, human mammary luminal epithelial cells; IL8, interleukin-8; $\mathrm{ION}$, ionomycin; KD, knockdown; MDM2, mouse double minute 2; NFAT, nuclear factor of activated T cells; OE, overexpression; PKA, protein kinase A; PKB, protein kinase B; Stat5, signal transducer and activator of transcription 5.

\section{ACKNOWLEDGMENTS AND FUNDING}

This work was supported by the National Institutes of Health (NIH) grants R01 CA112029, R01 CA121211, and R01 CA186662 (to RZ). The contents are solely the responsibility of the authors, and do not necessarily represent the official views of the National Institutes of Health. This work was also supported by the American Cancer Society (ACS) grant RSG-15-009-01-CDD (to WW). This work was also supported by the Natural Nature Science Foundation of China (81125020 to HW; 81230090 to WDZ). We thank Drs. Lei Shan, Hui-Zi Jin, Juan Su, and Shou-De Zhang for excellent assistance in the preparation of test compound.

\section{CONFLICTS OF INTEREST}

The authors declare that they have no competing financial interests.

\section{REFERENCES}

1. Pagliarini R, Shao W, Sellers WR. Oncogene addiction: pathways of therapeutic response, resistance, and road maps toward a cure. EMBO Rep. 2015; 16:280-296.

2. Torti D, Trusolino L. Oncogene addiction as a foundational rationale for targeted anti-cancer therapy: promises and perils. EMBO Mol Med. 2011; 3:623-636.

3. Santarius T, Shipley J, Brewer D, Stratton MR, Cooper CS. A census of amplified and overexpressed human cancer genes. Nat Rev Cancer. 2010; 10:59-64.

4. Wade M, Li YC, Wahl GM. MDM2, MDMX and p53 in oncogenesis and cancer therapy. Nat Rev Cancer. 2013; 13:83-96.

5. Nag S, Qin J, Srivenugopal KS, Wang M, Zhang R. The MDM2-p53 pathway revisited. J Biomed Res. 2013; 27:254-271.

6. Bouska A, Eischen CM. Murine double minute 2: p53independent roads lead to genome instability or death. Trends Biochem Sci. 2009; 34:279-286.

7. Qin JJ, Nag S, Voruganti S, Wang W, Zhang R. Natural product MDM2 inhibitors: Anticancer activity and mechanisms of action. Curr Med Chem. 2012; 19:5705-5725.

8. Vassilev LT, Vu BT, Graves B, Carvajal D, Podlaski F, Filipovic Z, Kong N, Kammlott U, Lukacs C, Klein C, Fotouhi N, Liu EA. In vivo activation of the p53 pathway by small-molecule antagonists of MDM2. Science. 2004; 303:844-848.

9. Issaeva N, Bozko P, Enge M, Protopopova M, Verhoef LG, Masucci M, Pramanik A, Selivanova G. Small molecule RITA binds to p53, blocks p53-HDM-2 interaction and activates p53 function in tumors. Nat Med. 2004; 10:1321-1328.

10. Shangary S, Qin D, McEachern D, Liu M, Miller RS, Qiu S, Nikolovska-Coleska Z, Ding K, Wang G, Chen J, Bernard D, Zhang J, Lu Y, et al. Temporal activation of p53 by a specific MDM2 inhibitor is selectively toxic to tumors and leads to complete tumor growth inhibition. Proc Natl Acad Sci U S A. 2008; 105:3933-3938.

11. Wang W, Qin JJ, Voruganti S, Srivenugopal KS, Nag S, Patil S, Sharma H, Wang MH, Wang H, Buolamwini JK, Zhang R. The pyrido[b]indole MDM2 inhibitor SP-141 exerts potent therapeutic effects in breast cancer models. Nat Commun. 2014; 5:5086.

12. Wang W, Qin JJ, Voruganti S, Wang MH, Sharma H, Patil S, Zhou J, Wang H, Mukhopadhyay D, Buolamwini JK, Zhang R. Identification of a new class of MDM2 inhibitor that inhibits growth of orthotopic pancreatic tumors in mice. Gastroenterology. 2014; 147:893-902. 
13. Qin JJ, Wang W, Voruganti S, Wang H, Zhang WD, Zhang R. Identification of a new class of natural product MDM2 inhibitor: In vitro and in vivo anti-breast cancer activities and target validation. Oncotarget. 2015; 6:2623-2640.

14. Ding Q, Zhang Z, Liu JJ, Jiang N, Zhang J, Ross TM, Chu XJ, Bartkovitz D, Podlaski F, Janson C, Tovar C, Filipovic ZM, Higgins B, et al. Discovery of RG7388, a potent and selective p53-MDM2 inhibitor in clinical development. J Med Chem. 2013; 56:5979-5983.

15. Rew Y, Sun D. Discovery of a small molecule MDM2 inhibitor (AMG 232) for treating cancer. J Med Chem. 2014; 57:6332-6341.

16. Qin JJ, Jin HZ, Fu JJ, Hu XJ, Wang Y, Yan SK, Zhang WD. Japonicones A-D, bioactive dimeric sesquiterpenes from Inula japonica Thunb. Bioorg Med Chem Lett. 2009; 19:710-713.

17. Qin JJ, Jin HZ, Huang Y, Zhang SD, Shan L, Voruganti S, Nag S, Wang W, Zhang WD, Zhang R. Selective cytotoxicity, inhibition of cell cycle progression, and induction of apoptosis in human breast cancer cells by sesquiterpenoids from Inula lineariifolia Turcz. Eur J Med Chem. 2013; 68:473-481.

18. Hu Z, Qin J, Zhang H, Wang D, Hua Y, Ding J, Shan L, Jin H, Zhang J, Zhang W. Japonicone A antagonizes the activity of TNF-alpha by directly targeting this cytokine and selectively disrupting its interaction with TNF receptor-1. Biochem Pharmacol. 2012; 84:1482-1491.

19. Wang GW, Qin JJ, Cheng XR, Shen YH, Shan L, Jin HZ, Zhang WD. Inula sesquiterpenoids: structural diversity, cytotoxicity and anti-tumor activity. Expert Opin Investig Drugs. 2014; 23:317-345.

20. Li X, Yang X, Liu Y, Gong N, Yao W, Chen P, Qin J, Jin H, Li J, Chu R, Shan L, Zhang R, Zhang W, et al. Japonicone A suppresses growth of Burkitt lymphoma cells through its effect on NF-kappaB. Clin Cancer Res. 2013; 19:2917-2928.

21. Du Y, Gong J, Tian X, Yan X, Guo T, Huang M, Zhang B, Hu X, Liu H, Wang Y, Li J, Li M. Japonicone A inhibits the growth of non-small cell lung cancer cells via mitochondria-mediated pathways. Tumour Biol. 2015.

22. Muller MR, Rao A. NFAT, immunity and cancer: a transcription factor comes of age. Nat Rev Immunol. 2010; 10:645-656.

23. Fric J, Zelante T, Wong AY, Mertes A, Yu HB, RicciardiCastagnoli P. NFAT control of innate immunity. Blood. 2012; 120:1380-1389.

24. Qin JJ, Nag S, Wang W, Zhou J, Zhang WD, Wang H, Zhang R. NFAT as cancer target: Mission possible? Biochim Biophys Acta. 2014; 1846:297-311.

25. Pan MG, Xiong Y, Chen F. NFAT gene family in inflammation and cancer. Curr Mol Med. 2013; 13:543-554.

26. Mancini M, Toker A. NFAT proteins: emerging roles in cancer progression. Nat Rev Cancer. 2009; 9:810-820.
27. Gaudineau B, Fougere M, Guaddachi F, Lemoine F, de la Grange P, Jauliac S. Lipocalin 2, the TNF-like receptor TWEAKR and its ligand TWEAK act downstream of NFAT1 to regulate breast cancer cell invasion. J Cell Sci. 2012; 125:4475-4486.

28. Zheng J, Fang F, Zeng X, Medler TR, Fiorillo AA, Clevenger CV. Negative cross talk between NFAT1 and Stat5 signaling in breast cancer. Mol Endocrinol. 2011; 25:2054-2064.

29. Pham LV, Tamayo AT, Yoshimura LC, Lin-Lee YC, Ford RJ. Constitutive NF-kappaB and NFAT activation in aggressive B-cell lymphomas synergistically activates the CD154 gene and maintains lymphoma cell survival. Blood. 2005; 106:3940-3947.

30. Yoeli-Lerner M, Yiu GK, Rabinovitz I, Erhardt P, Jauliac S, Toker A. Akt blocks breast cancer cell motility and invasion through the transcription factor NFAT. Mol Cell. 2005; 20:539-550.

31. Zhang X, Zhang Z, Cheng J, Li M, Wang W, Xu W, Wang H, Zhang R. Transcription factor NFAT1 activates the $\mathrm{mdm} 2$ oncogene independent of p53. J Biol Chem. 2012; 287:30468-30476.

32. Buchholz M, Schatz A, Wagner M, Michl P, Linhart T, Adler G, Gress TM, Ellenrieder V. Overexpression of c-myc in pancreatic cancer caused by ectopic activation of NFATc1 and the Ca2+/calcineurin signaling pathway. EMBO J. 2006; 25:3714-3724.

33. Mognol GP, de Araujo-Souza PS, Robbs BK, Teixeira LK, Viola JP. Transcriptional regulation of the c-Myc promoter by NFAT1 involves negative and positive NFAT-responsive elements. Cell Cycle. 2012; 11:1014-1028.

34. Yiu GK, Toker A. NFAT induces breast cancer cell invasion by promoting the induction of cyclooxygenase-2. J Biol Chem. 2006; 281:12210-12217.

35. Yiu GK, Kaunisto A, Chin YR, Toker A. NFAT promotes carcinoma invasive migration through glypican-6. Biochem J. 2011; 440:157-166.

36. Yoeli-Lerner M, Chin YR, Hansen CK, Toker A. Akt/ protein kinase $b$ and glycogen synthase kinase-3beta signaling pathway regulates cell migration through the NFAT1 transcription factor. Mol Cancer Res. 2009; 7:425-432.

37. Kaunisto A, Henry WS, Montaser-Kouhsari L, Jaminet SC, Oh EY, Zhao L, Luo HR, Beck AH, Toker A. NFAT1 promotes intratumoral neutrophil infiltration by regulating IL8 expression in breast cancer. Mol Oncol. 2015; 9:1140-1154.

38. Quang CT, Leboucher S, Passaro D, Fuhrmann L, Nourieh M, Vincent-Salomon A, Ghysdael J. The calcineurin/NFAT pathway is activated in diagnostic breast cancer cases and is essential to survival and metastasis of mammary cancer cells. Cell Death Dis. 2015; 6:e1658.

39. Yu H, van Berkel TJ, Biessen EA. Therapeutic potential of VIVIT, a selective peptide inhibitor of nuclear factor of activated $\mathrm{T}$ cells, in cardiovascular disorders. Cardiovasc Drug Rev. 2007; 25:175-187. 
40. Elloumi HZ, Maharshak N, Rao KN, Kobayashi T, Ryu HS, Muhlbauer M, Li F, Jobin C, Plevy SE. A cell permeable peptide inhibitor of NFAT inhibits macrophage cytokine expression and ameliorates experimental colitis. PLoS One. 2012; 7:e34172.

41. Aramburu J, Yaffe MB, Lopez-Rodriguez C, Cantley LC, Hogan PG, Rao A. Affinity-driven peptide selection of an NFAT inhibitor more selective than cyclosporin A. Science. 1999; 285:2129-2133.

42. Lee CR, Chun JN, Kim SY, Park S, Kim SH, Park EJ, Kim IS, Cho NH, Kim IG, So I, Kim TW, Jeon JH. Cyclosporin A suppresses prostate cancer cell growth through CaMKKbeta/AMPK-mediated inhibition of mTORC1 signaling. Biochem Pharmacol. 2012; 84:425-431.

43. Masuo T, Okamura S, Zhang Y, Mori M. Cyclosporine A inhibits colorectal cancer proliferation probably by regulating expression levels of c-Myc, p21(WAF1/CIP1) and proliferating cell nuclear antigen. Cancer Lett. 2009; 285:66-72.

44. Kawahara T, Kashiwagi E, Ide H, Li Y, Zheng Y, Miyamoto Y, Netto GJ, Ishiguro H, Miyamoto H. Cyclosporine A and tacrolimus inhibit bladder cancer growth through down-regulation of NFATc1. Oncotarget. 2015; 6:1582-1593.

45. Garrido W, Munoz M, San Martin R, Quezada C. FK506 confers chemosensitivity to anticancer drugs in glioblastoma multiforme cells by decreasing the expression of the multiple resistance-associated protein-1. Biochem Biophys Res Commun. 2011; 411:62-68.

46. Sieber M, Karanik M, Brandt C, Blex C, Podtschaske M, Erdmann F, Rost R, Serfling E, Liebscher J, Patzel M, Radbruch A, Fischer G, Baumgrass R. Inhibition of calcineurin-NFAT signaling by the pyrazolopyrimidine compound NCI3. Eur J Immunol. 2007; 37:2617-2626.

47. Tie X, Han S, Meng L, Wang Y, Wu A. NFAT1 is highly expressed in, and regulates the invasion of, glioblastoma multiforme cells. PLoS One. 2013; 8:e66008.

48. Baumgart S, Chen NM, Siveke JT, Konig A, Zhang JS, Singh SK, Wolf E, Bartkuhn M, Esposito I, Hessmann E, Reinecke J, Nikorowitsch J, Brunner M, et al. Inflammationinduced NFATc1-STAT3 transcription complex promotes pancreatic cancer initiation by KrasG12D. Cancer Discov. 2014; 4:688-701.

49. Oikawa T, Nakamura A, Onishi N, Yamada T, Matsuo K, Saya H. Acquired expression of NFATc1 downregulates E-cadherin and promotes cancer cell invasion. Cancer Res. 2013; 73:5100-5109.

50. Fougere M, Gaudineau B, Barbier J, Guaddachi F, Feugeas JP, Auboeuf D, Jauliac S. NFAT3 transcription factor inhibits breast cancer cell motility by targeting the Lipocalin 2 gene. Oncogene. 2010; 29:2292-2301.
51. Berges C, Fuchs D, Opelz G, Daniel V, Naujokat C. Helenalin suppresses essential immune functions of activated CD4+ T cells by multiple mechanisms. Mol Immunol. 2009; 46:2892-2901.

52. Singh SK, Baumgart S, Singh G, Konig AO, Reutlinger K, Hofbauer LC, Barth P, Gress TM, Lomberk G, Urrutia R, Fernandez-Zapico ME, Ellenrieder V. Disruption of a nuclear NFATc2 protein stabilization loop confers breast and pancreatic cancer growth suppression by zoledronic acid. J Biol Chem. 2011; 286:28761-28771.

53. Marquez N, Sancho R, Ballero M, Bremner P, Appendino G, Fiebich BL, Heinrich M, Munoz E. Imperatorin inhibits T-cell proliferation by targeting the transcription factor NFAT. Planta Med. 2004; 70:1016-1021.

54. Yang QF, Dalgard CL, Eidelman O, Jozwik C, Pollard BS, Srivastava M, Pollard HB. Digitoxin induces apoptosis in cancer cells by inhibiting nuclear factor of activated T-cells-driven c-MYC expression. J Carcinog. $2013 ; 12: 8$.

55. Wang W, Rayburn ER, Velu SE, Nadkarni DH, Murugesan S, Zhang R. In vitro and in vivo anticancer activity of novel synthetic makaluvamine analogues. Clin Cancer Res. 2009; 15:3511-3518.

56. Chen D, Zhang Z, Li M, Wang W, Li Y, Rayburn ER, Hill DL, Wang H, Zhang R. Ribosomal protein S7 as a novel modulator of p53-MDM2 interaction: binding to MDM2, stabilization of $\mathrm{p} 53$ protein, and activation of $\mathrm{p} 53$ function. Oncogene. 2007; 26:5029-5037.

57. Wang W, Cheng J, Qin JJ, Voruganti S, Nag S, Fan J, Gao Q, Zhang R. RYBP expression is associated with better survival of patients with hepatocellular carcinoma (HCC) and responsiveness to chemotherapy of HCC cells in vitro and in vivo. Oncotarget. 2014; 5:11604-11619.

58. Voruganti S, Qin JJ, Sarkar S, Nag S, Walbi IA, Wang S, Zhao Y, Wang W, Zhang R. Oral nano-delivery of anticancer ginsenoside 25-OCH3-PPD, a natural inhibitor of the MDM2 oncogene: Nanoparticle preparation, characterization, in vitro and in vivo anti-prostate cancer activity, and mechanisms of action. Oncotarget. 2015; 6:21379-21394.

59. Wang W, Zhang X, Qin JJ, Voruganti S, Nag SA, Wang $\mathrm{MH}$, Wang $\mathrm{H}$, Zhang R. Natural product ginsenoside 25-OCH3-PPD inhibits breast cancer growth and metastasis through down-regulating MDM2. PLoS One. 2012; 7:e41586.

60. Yang X, Wang W, Qin JJ, Wang MH, Sharma H, Buolamwini JK, Wang H, Zhang R. JKA97, a novel benzylidene analog of harmine, exerts anti-cancer effects by inducing G1 arrest, apoptosis, and p53-independent up-regulation of p21. PLoS One. 2012; 7:e34303. 